\title{
Primeiros registros de Climacia (Neuroptera: Sisyridae) para o estado de Roraima, Brasil
}

\author{
Emely Trajano de Menezes ${ }^{1}$; Ismael Barreto de Oliveira ${ }^{2}$; Rafael Boldrini ${ }^{3}$; Bianca \\ Maíra de Paiva Ottoni Boldrini ${ }^{4}$ \\ ${ }^{1,2,3}$ UFRR - Universidade Federal de Roraima, Centro de Estudos da Biodiversidade (CBio), Campus Parica- \\ rana, CEP 69310-000, Boa Vista, Roraima, Brasil; E-mail: etm13emely@gmail.com; ismaelbdo@gmail.com²; \\ rafaelboldrini.2@gmail.com \\ ${ }^{4}$ UFRR - Universidade Federal de Roraima, Colégio de Aplicação (CAp), Campus Paricarana, CEP 69310-000, Boa \\ Vista, Roraima, Brasil; E-mail: biancaottoni@gmail.com.
}

Recebido em: 14 de maio de 2018. Aceito em: 6 de julho de 2018. Publicado PDF em: 18 de julho de 2018

\section{RESUMO}

Primeiros registros de Climacia (Neuroptera: Sisyridae) para o estado de Roraima, Brasil. A família Sisyridae é a única exclusivamente aquática da ordem Neuroptera no Brasil. Este trabalho apresenta os primeiros registros de espécies do gênero Climacia McLachlan para o estado de Roraima. Os espécimes adultos foram coletados utilizando armadilhas do tipo lençol iluminado e Pennsylvania. Em seguida, os espécimes foram fixados em etanol $80 \%$ e transportados ao Laboratório de Entomologia da Universidade Federal de Roraima (UFRR), onde ocorreu triagem e identificação utilizando estereomicroscópio. No estado de Roraima havia apenas o registro de Sisyra. Como resultados neste trabalho, encontraram-se quatro espécies para o gênero Climacia (C. basalis Banks, C. nota Parfin \& Gurney, C. punctulata Flint e C. townesi Parfin \& Gurney), que constituem, portanto, os primeiros registros para o estado de Roraima. Além disso, a espécie C. basalis tem seu primeiro registro para a região Norte do Brasil.

PALAVRAS CHAVE: Neotropical, Sisyridae, Taxonomia, Entomofauna, Biodiversidade.

\begin{abstract}
First records of Climacia (Neuroptera: Sisyridae) to state of Roraima, Brazil. The Sisyridae family is the only one exclusively aquatic of the order Neuroptera in Brazil. This work presents the first records of species of genus Climacia McLachlan in the state of Roraima. Adult specimens were collected using lighted sheet and Pennsylvania traps, fixed in $80 \%$ ethanol and transported to the Entomology Laboratory of the Federal University of Roraima (UFRR), where stereomicroscope screening and species identification were performed using taxonomic keys. In the state of Roraima there was only the record of the genus Sisyra. As results of this work, four species for the genus Climacia (C. basalis Banks, C. nota Parfin \& Gurney, C. punctulata Flint e C. townesi Parfin \& Gurney) were found, therefore they constitute the first records in the state. In addition, C. basalis species has its first record to North Brazil.
\end{abstract}

KEY WORDS: Neotropical, Sisyridae, Taxonomy, Entomofauna, Biodiversity. 


\section{INTRODUÇÃO}

A família Sisyridae é a única exclusivamente aquática da ordem Neuroptera no Brasil. As fêmeas adultas depositam seus ovos em esponjas de água doce, na qual as larvas em sua fase aquática se alimentam do conteúdo das células das esponjas e irão permanecer até seu último estágio de vida larval, por isso é considerada um parasitismo (Penny, 1981; Melão \& Rocha, 1996).

Essa família é composta por quatro gêneros (Sisyrina Banks, Sisyborina Montserrat, Sisyra Burmeister e Climacia McLachlan). No Brasil, ocorrem dois desses gêneros: Sisyra possuindo distribuição mundial e Climacia restrita para as regiões Neotropical e Neártica.

O gênero Climacia possui 21 espécies relatadas no Neotrópico, dentre elas 10 ocorrem no Brasil (Parfin \& Gurney, 1956; Assmar \& Salles, 2017). Segundo Assmar \& Salles (2017), para a região norte há registros desse grupo para os estados do Amazonas ( $C$. negrensis; $C$. bimaculata e $C$. townesi), Acre (C. townesi), Pará $(C$. nota) e Rondônia $(C$. punctulata).

O estado de Roraima se localiza ao Norte do Brasil e os estudos para essa família no estado ainda são escassos. Por isso, o presente estudo apresenta os primeiros registros de espécies do gênero Climacia para o estado de Roraima e $C$. basalis para o Norte do Brasil.

\section{MATERIAL E MÉTODOS}

Nos meses de junho, agosto e dezembro de 2017, foram capturados adultos através de armadilha do tipo lençol iluminado, com lâmpada de luz mista de $250 \mathrm{w}$ e luz negra de LED. Além disso, também foi utilizada armadilha luminosa tipo Pennsylvania. Os espécimes foram coletados nas localidades: campus Cauamé da UFRR (0251'47,76"N/ 60 42'53,89"W), município de Boa Vista, no Rio Arraia $\quad\left(03^{\circ} 21^{\prime} 20,43^{\prime \prime N} / \quad 59^{\circ} 54^{\prime} 11,67^{\prime \prime} \mathrm{W}\right)$, município de Bonfim, e Floresta Nacional de Roraima (FLONA de Roraima) $\left(02^{\circ}\right.$ $\left.56^{\prime} 18,05^{\prime \prime} \mathrm{N} / 61^{\circ} 37^{\prime} 27,28^{\prime \prime} \mathrm{W}\right)$, município do Alto Alegre (Fig. 1), foram fixados em álcool $80 \%$ e transportados ao Laboratório de Entomologia da UFRR. No laboratório, ocorreu a triagem utilizando estereomicroscópio e a identificação e diagnose seguiu Parfin \& Gurney (1956), Penny (1981) e Assmar \&
Salles (2017). O mapa com as localidades de coletas foi produzido através do software QGis versão 2.18 Las Palmas (Fig. 1). Ainda, foram feitas fotografias dos espécimes representantes de cada espécie (Fig. 2A-D), bem como o padrão de venação (Fig. 3A-D) com câmera Bioptika CMOS 10 megapixels acoplada em um estereomicroscópio Olympus SZ51 e ao microscópio óptico Nikon Eclipse E200. Os materiais examinados estão depositados na coleção do Laboratório de Entomologia da UFRR.

\section{RESULTADOS}

Climacia basalis Banks, 1913

(Fig. 2A e Fig. 3A)

Diagnose:

Imago macho: Corpo amarelo pálido, antenas com os primeiros segmentos basais marrom amarelados e os outros castanhos escuros a pretos; cabeça e tórax com manchas castanho claros e abdômen amarelo pálido; pronoto com estrias marrom médio; asas amarelas, com uma linha radiomedial castanha escura se estendendo longitudinalmente no setor radial. Presença de manchas marrom escuras na asa anterior entre as veias longitudinais e poucas manchas no final destas veias, e presença de pequena faixa marrom escura na região anal. Asa posterior com uma faixa acastanhada na região pterostigmal. Genitália macho: Segmentos abdominais levemente esclerosados. Oitavo segmento mais longo ventralmente com numerosas cerdas. Nono segmento menor que o oitavo, com longas cerdas dentígeras posteroventralmente. Nono gonocoxito (ectoprocto) com ápice arredondado, subtriangular, pontiagudo dorsalmente, com numerosas cerdas longas baseadas em dentígeros (Assmar \& Salles, 2017).

Distribuição: Brasil e Guiana (Flint, 2006). No Brasil há registros para os estados do Espírito Santo e Mato Grosso do Sul (Assmar \& Salles, 2017). Novo registro: estado de Roraima.

Observações: Esta espécie foi registrada primeiramente no Brasil para o estado de Mato Grosso do Sul por Navás em 1933 como $C$. desordenata e porteriormente em 1935 como $C$. basalis segundo Assmar \& Salles (2017). O trabalho de Monserrat (2005) apresenta a mesma espécie $C$. desordenata como um novo nome para $C$. basalis para haver uma 


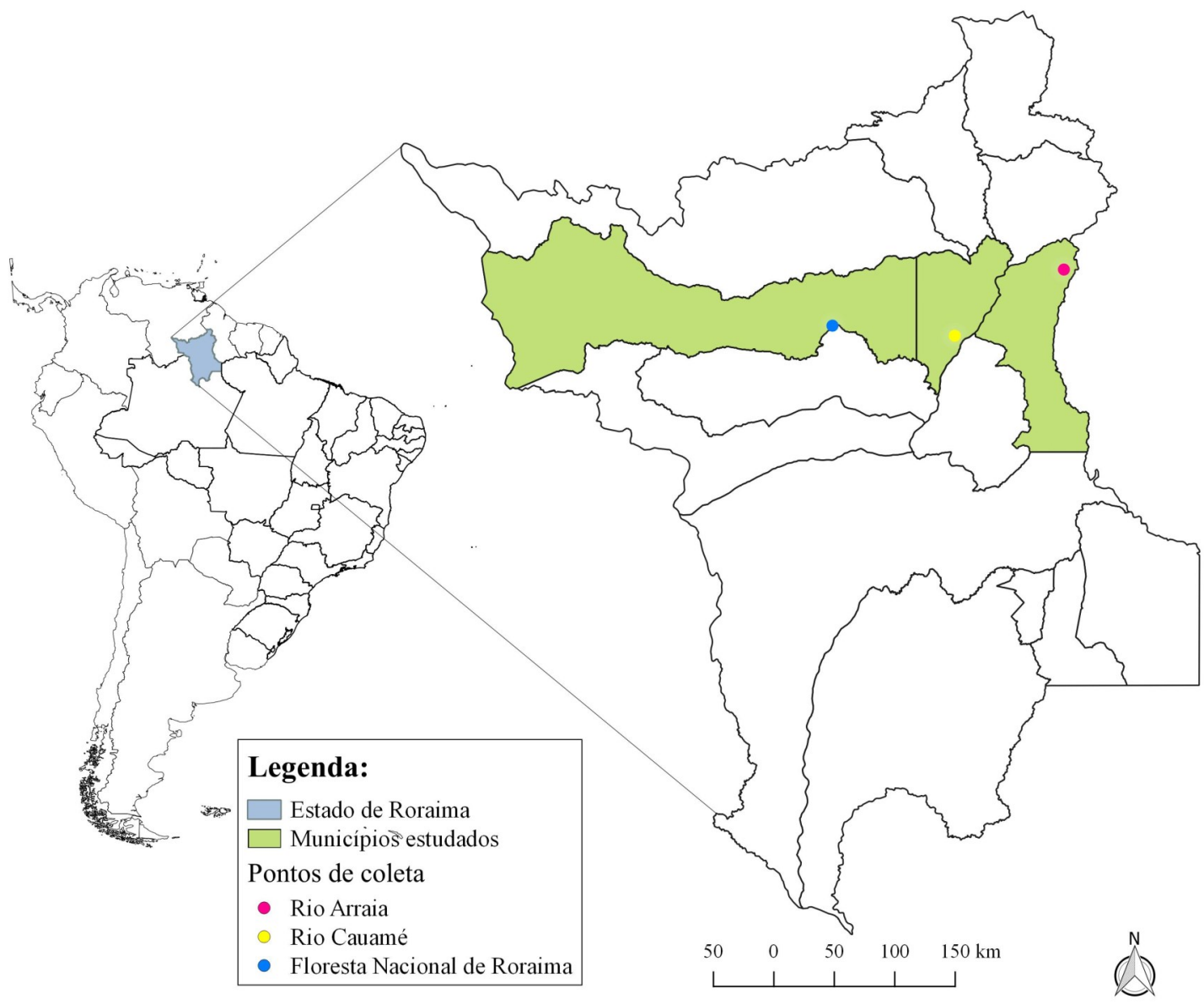

Figura 1. Locais no estado de Roraima onde espécimes de Climacia (Neuroptera: Sisyridae) foram coletados.

homonímia, no entanto ambas podem ser consideradas como mesma espécie com pouco grau de variação apresentada nas manchas das asas. Nos espécimes coletados no presente estudo, as asas anteriores apresentaram padrão de coloração semelhante a $C$. desordenata, e a descrição da genitália conforme as figuras no trabalho de Assmar e Salles (2017).

Material examinado: três machos e três fêmeas, Brasil, Roraima, Boa Vista, UFRR Campus Cauamé, Rio Cauamé, 02 $51^{\prime} 47,76^{\prime \prime N} /$ 6042'53,89"W, 10.vi.2017, armadilha luminosa lençol iluminado com luz fluorescente e luz negra de LED, Boldrini, R.; Oliveira, I. B.; Menezes, E. T.; Barbosa, F.S.; Almeida, M.L.S.; Picanço, M. M. col.

Climacia nota Parfin \& Gurney, 1956

(Fig. 2B e Fig. 3B)

Diagnose:

Imago fêmea: Cabeça com vértice amarelo brilhante; labro acastanhado para margem anterior; palpo amarelo; segmento basal da antena amarelo, levemente acastanhado dorsolateralmente para o segundo segmento; segundo para quarto (aproximadamente) segmentos marrons amarelados, mais claros que os 20 segmentos que são marrons escuros, e os próximos 20 segmentos são mais claros. Pernas amarelas; pronoto amarelado dorsalmente, quase tão longo quanto largo, com listras escuras irregulares, mesonoto e metanoto amarelado, mais escuro lateralmente, pleura acastanhada. Abdômen amarelado (Parfin \& Gurney, 1956).

Distribuição: Bolívia, Brasil, Guiana e Venezuela (Flint, 2006). No Brasil há registro apenas para o estado do Pará (Assmar \& Salles, 2017). Novo registro: estado de Roraima.

Material examinado: seis fêmeas, Brasil, Roraima, Bonfim, Rio Arraia, 03 $21^{\prime} 20,43^{\prime \prime N} /$ 5954'11,67"W， 29.viii.2017, armadilha luminosa Pennsylvania, Boldrini, R.; Oliveira, I. B.; Barbosa, F.S. col. 

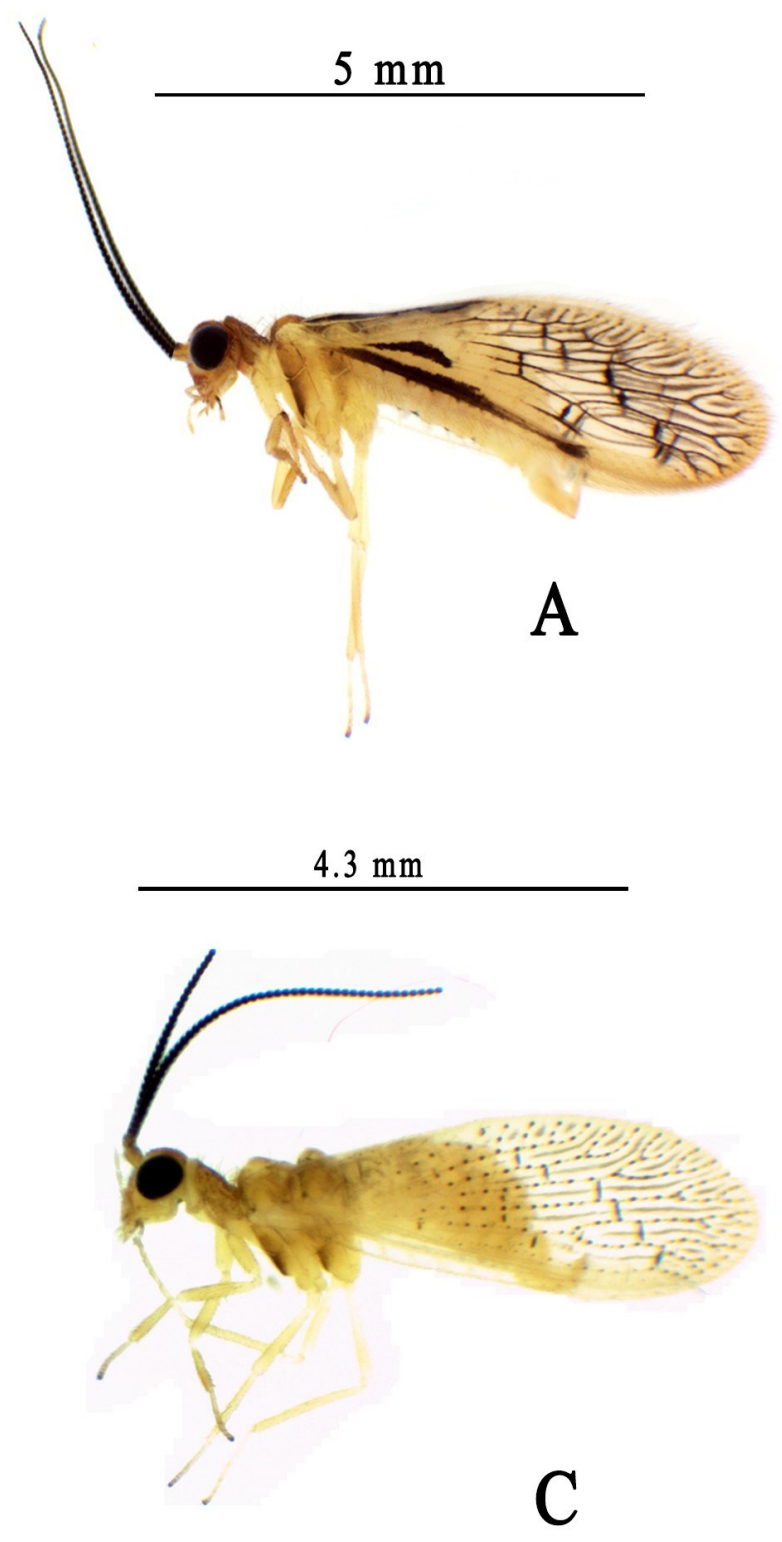
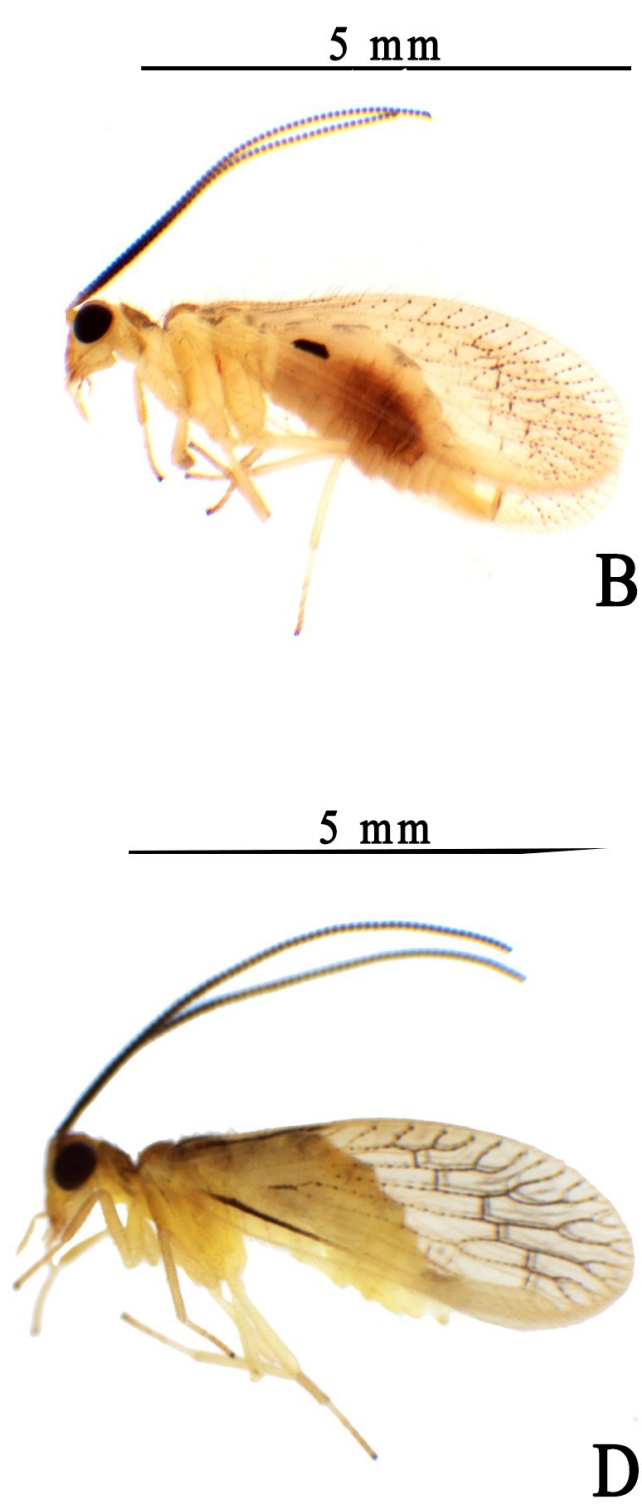

Figura 2. Adultos do gênero Climacia McLachlan para o estado de Roraima, vista lateral. A. C. basalis Banks; B. C. nota Parfin \& Gurney; C. C. punctulata Flint; D. C. townesi Parfin \& Gurney.

Climacia punctulata Flint, 2006

(Fig. 2C e Fig. 3C)

Diagnose:

Imago fêmea: Cabeça, tórax e apêndices pálidos, marrom amarelados, com faixa marrom mais escura na cabeça e no pronoto, tornando-se mais ampla e difusa no meso e metanoto. Antena com escapo levemente escurecido, segmentos flagelares basais marrom-claro, segmentos além do apical progressivamente mais escuro.
Distribuição: Brasil (Flint, 2006). No Brasil há registros para os estados Espírito Santo, Minas Gerais e Rondônia (Assmar \& Salles, 2017). Novo registro: estado de Roraima.

Observações: Esta espécie foi registrada e descrita pela primeira vez por Flint (2006), as descrições foram somente para os espécimes machos encontrados. No presente trabalho foi encontrado no material apenas uma fêmea.

Material examinado: uma fêmea, Brasil, Roraima, Alto Alegre, Floresta Nacional de 

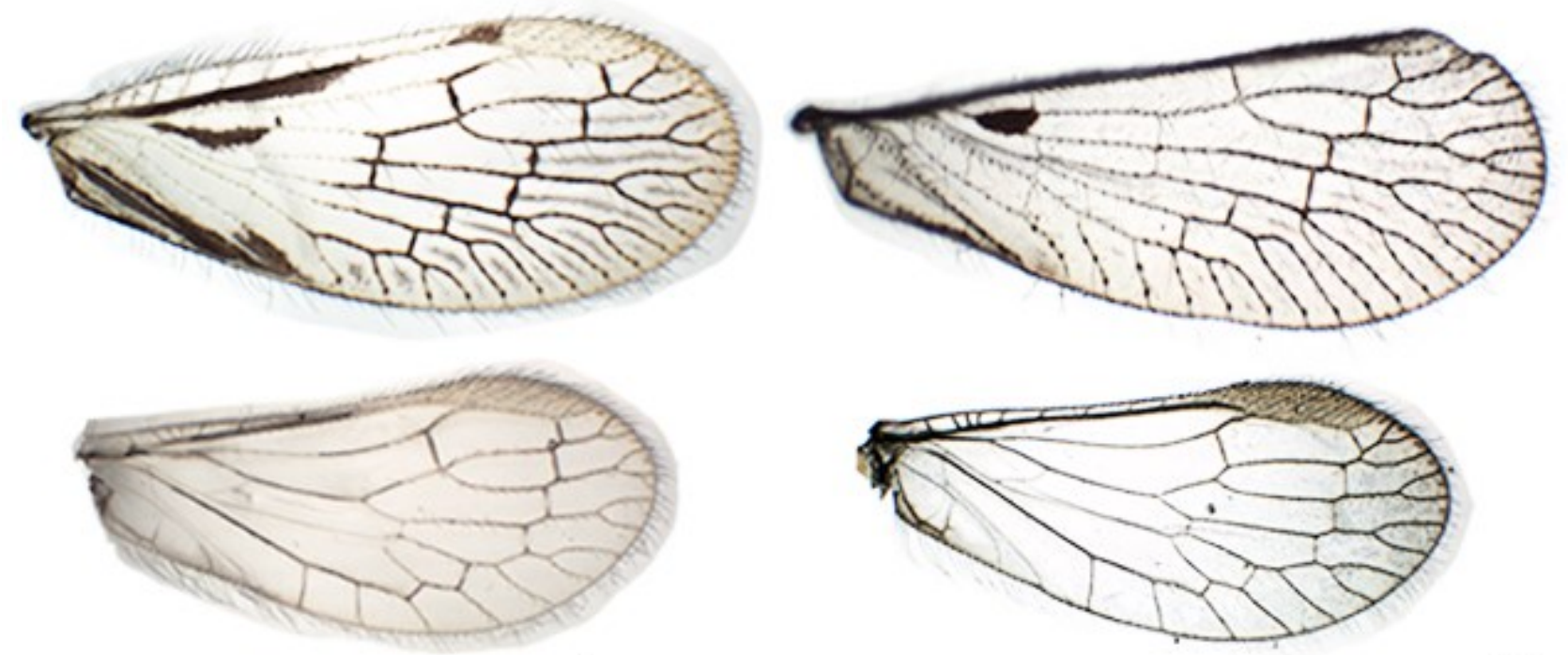

A

B
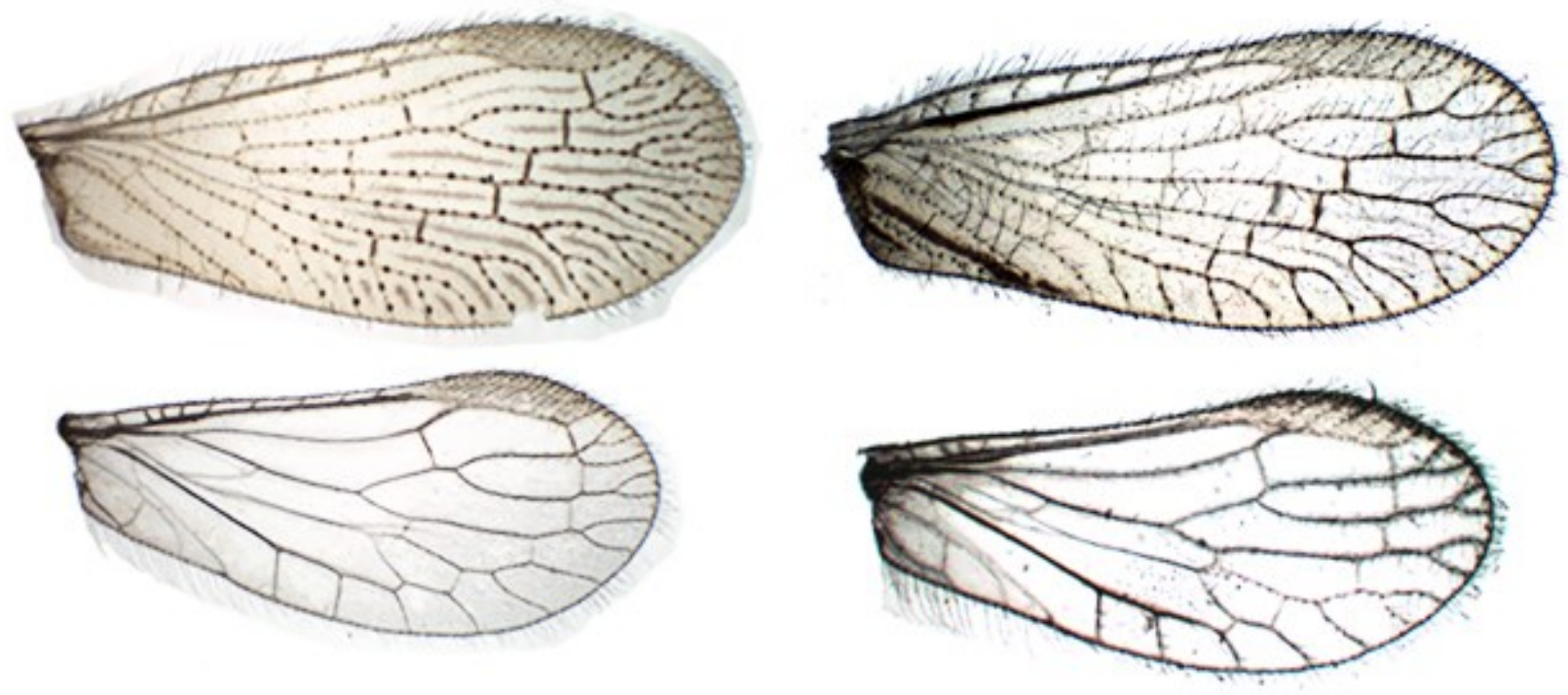

C

Figura 3. Asas de Climacia Mc Lachlan, asa anterior (superior) e asa posterior (inferior). A. $C$. basalis Banks; B. C. nota Parfin \& Gurney; C. C. punctulata Flint; D. C. townesi Parfin \& Gurney.

Roraima, $\quad 02^{\circ} 56^{\prime} 18,05^{\prime \prime} \mathrm{N} / \quad 61^{\circ} 37^{\prime} 27,28^{\prime \prime} \mathrm{W}$, 18.xii.2017, armadilha luminosa Pennsylvania, Boldrini, R., Oliveira, I.B., Barbosa, F.S., Almeida, M.L.S., Picanço, M.M. e Xavier, F.F. col.

Climacia townesi Parfin \& Gurney, 1956 (Fig. 2D e Fig. 3D)

Diagnose:
Imago macho: Corpo amarelo pálido, antenas com primeiro segmento basal marrom claro e os outros castanhos escuros a pretos. Cabeça e tórax com manchas castanho claros a médio e abdômen amarelo pálido; pronoto amarelado com riscas avermelhadas e castanho escuras; pernas amarelas com sombreado acastanhado nas coxas meso e metatorácicas; abdômen amarelado. Asas amarelas, com presença de manchas marrom escuras na asa anterior, 
subcostal e na região anal. Pernas amarelas com exceção do último segmento. Genitália do macho: Décimo esterno com anel caudal de cerdas longas pouco desenvolvidas. Lobo lateral do décimo esterno fortemente esclerosado, com cerdas longas com bases dentígeras. Nono esterno formando uma projeção medial, ventral e septiforme (Penny, 1981).

Distribuição: Brasil, Guiana, Peru e Venezuela (Flint, 2006). No Brasil há registros para os estados do Acre e Amazonas (Penny, 1981; Assmar \& Salles, 2017). Novo registro: estado de Roraima.

Observações: Esta espécie foi descrita originalmente por Parfin \& Gurney (1956) somente com os espécimes adultos fêmeas. Penny (1981) em seus registros, traz uma descrição para os machos complementando a certa identificação desta espécie. No presente trabalho, a espécie estudada apresenta uma variação no padrão de manchas das asas anteriores. Nas descrições originais dos autores anteriormente citados, há evidências das manchas somente na região anal, no entanto em todos os indivíduos analisados neste estudo apresentam padrão de mancha na região subcostal, além da anal. A descrição da genitália da espécie estudada mostra-se semelhante com o desenhado por Penny (Fig.12 de Penny, 1981).

Material examinado: oito machos e uma fêmea, Brasil, Roraima, Boa Vista, UFRR Campus Cauamé, Rio Cauamé, 02 $51^{\prime} 47,76^{\prime \prime N} /$ $60^{\circ} 42^{\prime} 53,89^{\prime \prime} \mathrm{W}, \quad$ 10.vi.2017, armadilha luminosa lençol iluminado com luz fluorescente e luz negra de LED, Boldrini, R.; Oliveira, I. B.; Menezes, E. T.; Barbosa, F.S.; Almeida, M.L.S.; Picanço, M. M. col.

\section{CONCLUSÃO}

Neste estudo temos os primeiros registros de Climacia para o estado de Roraima, considerando importante para futuros estudos ambientais e da biologia do grupo. As espécies C. basalis e C. townesi apresentaram variações nas manchas das asas anteriores com relação às descrições originais (Banks, 1913; Parfin \& Gurney, 1956; Penny, 1981; Monserrat, 2005 e Assmar \& Salles, 2017), e por outro lado, as genitálias não mostraram diferenças com as dos trabalhos citados. Nesse caso pode-se inferir às razões ambientais ou genéticas nessas espécies, visto que ambas foram coletadas no mesmo local. Vale ressaltar a importância da continuação dos estudos, sendo possível realizar também estudos moleculares com este grupo.

\section{AGRADECIMENTOS}

A rede BIONORTE projetos $\mathrm{CNPq}$ (Conselho Nacional de Desenvolvimento Científico e Tecnológico) PROC 407623/ 2013 -2 e 401784/ 2016-9 pelo apoio financeiro concedido. $\mathrm{E}$ as bolsas de Iniciação científica da Universidade Federal de Roraima e Programa de Iniciação Científica PIBIC/CNPq.

\section{REFERÊNCIAS BIBLIOGRÁFICAS}

Assmar, A.C. \& Salles, F.F. 2017. Taxonomic and distributional notes on Spongilla-flies (Neuroptera: Sisyridae) from Southeastern Brazil with first interactive key to the species of the country. Zootaxa 4273: 80-92.

Banks, N. 1913. New exotic neuropteroid insects. Proceedings of the Entomological Society of Washington 15: 137-142.

Flint, O. S. JR. 2006. New species and records of Neotropical Sisyridae with special reference to Sisyra (Insecta: Neuroptera). Proceedings of the Biological Society of Washington 119(2): 279-286.

Melão, M. G. G \& Rocha, O. 1996. Macrofauna associada a Metania spinata (Carter, 1881), Porifera, Metaniidae. Acta Liminologica Brasiliensia 8: 59-64.

Monserrat, V. J. 2005. Nuevos datos sobre algunas pequeñas familias de neurópteros (Insecta: Neuroptera: Nevrorthidae, Osmylidae, Sisyridae, Dilaridae). Heteropterus Revista de Entomologia 5: $1-26$.

Parfin, S.I. \& Gurney, A.B. 1956. The spongilla-flies, with special reference to those of the western hemisphere (Sisyridae, Neuroptera). Proceedings of the United States National Museum 105: 421-529 (www.doi.org/10.5479/si.00963801.105-3360.421). Acesso em 11/07/2017.

Penny, N. D. 1981, Neuroptera of the Amazon Basin. Part 1. Sisyridae. Acta Amazonica 11(1): 157-169. 